\title{
Heat transfer from convecting-radiating fin through optimized Chebyshev polynomials with interior point algorithm
}

https://doi.org/10.1515/nleng-2017-0163

Received Dec 7, 2017; revised Mar 3, 2018; accepted Mar 17, 2018.

\begin{abstract}
In this paper, the problem of determining heat transfer from convecting-radiating fin of triangular and concave parabolic shapes is investigated. We consider onedimensional, steady conduction in the fin and neglect radiative exchange between adjacent fins and between the fin and its primary surface. A novel intelligent computational approach is developed for searching the solution. In order to achieve this aim, the governing equation is transformed into an equivalent problem whose boundary conditions are such that they are convenient to apply reformed version of Chebyshev polynomials of the first kind. These Chebyshev polynomials based functions construct approximate series solution with unknown weights. The mathematical formulation of optimization problem consists of an unsupervised error which is minimized by tuning weights via interior point method. The trial approximate solution is validated by imposing tolerance constrained into optimization problem. Additionally, heat transfer rate and the fin efficiency are reported.
\end{abstract}

Keywords: Chebyshev polynomial of the first kind; Interior point method; Temperature distribution; Fin efficiency; Heat transfer rate

\footnotetext{
*Corresponding Author: Elyas Shivanian, Department of Applied Mathematics, Imam Khomeini International University, Qazvin, 34149-16818, Iran, E-mail: shivanian@sci.ikiu.ac.ir, Tel/Fax: +989126825371

Mahdi Keshtkar, Department of Applied Mathematics, Imam Khomeini International University, Qazvin, 34149-16818, Iran Hamidreza Navidi, Department of Applied Mathematics, Shahed University, P.O. Box 18155/159, Tehran, Iran
}

\section{Preliminaries and problem formulation}

The heat dissipation mechanism considered in literature is either pure convection or pure radiation. In applications where fins operate in a free or natural convection environment, the contribution of radiation is equally significant, and therefore the design must allow for occurring both convection and radiation. As an application, it can be mentioned to stamped heat sink or extruded heat sink designed for cooling a transistor. Even if forced convection is employed for cooling, radiation is significant if the operating temperatures are high as is the case with a finned regenerator $[1,2]$.

Enhancement of heat transfer employing fins is important in a multitude of heat exchange equipment [3-16]. It is clear from the literature review that the research has been greatly focused on the theoretical and experimental thermal analysis of both solid fins and porous fins with different profiles and thermophysical properties due to wide range of applications [17-34], also see the refs [35-38, 3844] to receive more information.

Furthermore, to see some very recent investigations on convective-radiative fin heat transfer, the interest readers are referred to [45-47]. Mosayebidorcheh et. al. have obtained an optimum design point for fin geometry so that heat transfer rate reaches to a maximum value in a constant fin volume [45]. In [46], authors have applied spectral collocation method for transient thermal analysis of coupled conductive, convective and radiative heat transfer in the moving plate with temperature dependent properties and heat generation. A spectral element method (SEM) has been developed in [47] in order to solve coupled conductive, convective and radiative heat transfer in moving porous fins of trapezoidal, convex parabolic and concave parabolic profiles.

We consider a longitudinal fin of arbitrary profile attached to a primary surface at temperature $T_{b}$. Let the fin length be $L$ and its thicknesses at the base and at the tip be $w_{b}$ and $w_{t}$, respectively. Further, let $w(x)$ represent the fin 
thickness at any distance $x$ (measured from the base). Both top and bottom faces of the fin interact with the surroundings through convection and radiation. The convection process is characterized by the heat transfer coefficient $h$ and the environment temperature $T_{\infty}$. To describe the surface radiation loss, we assume an emissivity $\varepsilon$ and an effective sink temperature $T_{s}$. Assuming one-dimensional conduction, constant thermal parameters, and neglecting finto-base and fin-to-fin radiation interaction, the governing equation for a unit depth of the fin is as follows [2]:

$$
\frac{\mathrm{d}}{\mathrm{d} X}\left[w(X) \frac{\mathrm{d} T}{\mathrm{~d} X}\right]=\frac{2 h}{k}\left(T-T_{\infty}\right)+\frac{2 \varepsilon \sigma}{k}\left(T^{4}-T_{s}^{4}\right),
$$

where $h, k, \varepsilon, \sigma, T_{\infty}$ and $T_{s}$ denote convective heat transfer coefficient, thermal conductivity of fin material, surface emissivity, Stefan-Boltzmann constant, environment temperature for convection and effective sink temperature for radiation, respectively. Introducing the dimensionless variables $\theta=\frac{T}{T_{b}}, \theta_{\infty}=\frac{T_{\infty}}{T_{b}}, \theta_{s}=\frac{T_{s}}{T_{b}}, x=\frac{X}{L}, \alpha=\frac{2 L}{w_{b}}$, $B i=\frac{h w_{b}}{2 k}$, and $N_{r}=\frac{\varepsilon \sigma w_{b} T_{b}^{3}}{2 k}$, Eq. (1) is converted to

$$
w(x) \frac{\mathrm{d}^{2} \theta}{\mathrm{d} x^{2}}+\frac{\mathrm{d} w}{\mathrm{~d} x} \frac{\mathrm{d} \theta}{\mathrm{d} x}=\alpha^{2} B i\left(\theta-\theta_{\infty}\right)+\alpha^{2} N_{r}\left(\theta^{4}-\theta_{s}^{4}\right) .
$$

To solve Eq. (2), the profile function $w(x)$ and the boundary conditions must be specified. The profile shapes chosen for the present work are triangular and concave parabolic. The fin base temperature in each case is assumed to be constant $T_{b}$. For the triangular and parabolic fins, the tip heat fluxes are obviously zero. Therefore

Triangular fin:

$$
\left.\theta\right|_{x=0}=1,\left.\quad \frac{\mathrm{d} \theta}{\mathrm{d} x}\right|_{x=1}=0, \quad w(x)=1-x,
$$

Concave parabolic fin:

$$
\left.\theta\right|_{x=0}=1,\left.\quad \frac{\mathrm{d} \theta}{\mathrm{d} x}\right|_{x=1}=0, \quad w(x)=1-x^{2} .
$$

The problem formulated by Eq. (2) with boundary conditions (3) and (4) have been investigated numerically and semi-analytically by many researchers, see $[2,48-51]$ and references therein.

In this article, we propose a new intelligent computational approach to obtain solution for the non-linear second-order boundary value problem (2)-(4). First, we transform the governing equation into an equivalent problem whose boundary conditions are $[-1,1]$. In this way, they are convenient to apply reformed version of Chebyshev polynomials of the first kind. Then we optimize Chebyshev polynomials of the first kind to construct approximate series solution with unknown weights. Furthermore, it is set up an optimization problem based on unsupervised error as objective function subject to a tolerance as constraint. This optimization problem is minimized by tuning weights via interior point method. This numerical based technique enables us to overcome the nonlinearity in the mentioned boundary value problem and then to obtain accurate solution. Moreover, in some exactly solvable cases, we compare the approximate solution with the exact one. Also, the fin efficiency and heat transfer rate with respect are reported.

\section{High order derivatives of basis functions}

Chebyshev polynomials [52] are very useful as orthogonal polynomials on the interval $[-1,1]$ of the real line. These polynomials have very good properties in the approximation of functions so that appear frequently in several fields of mathematics, physics and engineering.

\subsection{Basic properties of Chebyshev polynomials}

The Chebyshev polynomials of the first kind, known as $T_{n}(x)=\cos (n \arccos x)$, can be obtained by means of Rodrigue's formula [53]

$$
\begin{aligned}
T_{n}(x) & =\frac{\Gamma\left(\frac{1}{2}\right)}{(-2)^{n} \Gamma\left(n+\frac{1}{2}\right)} \sqrt{1-x^{2}} \frac{\mathrm{d}^{n}}{\mathrm{~d} x^{n}}\left(1-x^{2}\right)^{n-\frac{1}{2}}, \\
n & =0,1,2, \cdots .
\end{aligned}
$$

The Chebyshev polynomials of the first kind can be developed by means of the generating function too, as follows:

$$
\frac{1-t x}{1-2 t x+t^{2}}=\sum_{n=0}^{+\infty} T_{n}(x) t^{n} .
$$

The first two Chebyshev polynomials $T_{n}(x)=1$ and $T_{n}(x)=x$ are known from (5), all other polynomials $T_{n}(x), n \geq 2$ can be obtained by means of the recurrence formula

$$
T_{n+1}(x)=2 x T_{n}(x)-T_{n-1}(x) .
$$

The derivative of $T_{n}(x)$ with respect to $x$ can be obtained from

$$
\begin{aligned}
& \left(1-x^{2}\right) T_{n}^{\prime}(x)=-n x T_{n}(x)+n T_{n-1}(x), x \neq \pm 1, \\
& T_{n}^{\prime}(-1)=n^{2}(-1)^{n+1}, \quad T_{n}^{\prime}(1)=n^{2}
\end{aligned}
$$


The following special values and properties of $T_{n}(x)$ are well established and will be useful:

$$
\begin{aligned}
& T_{n}(-x)=(-1)^{n} T_{n}(x), T_{n}(1)=1, T_{n}(-1)=(-1)^{n}, \\
& T_{2 n}(0)=(-1)^{n}, T_{2 n+1}(0)=0 .
\end{aligned}
$$

We can determine the orthogonality properties for the Chebyshev polynomials of the first kind from our knowledge of the orthogonality of the cosine functions, as

$$
\int_{-1}^{1} \frac{T_{n}(x) T_{m}(x)}{\sqrt{1-x^{2}}} \mathrm{~d} x= \begin{cases}0, & m \neq n \\ \frac{\pi}{2}, & m=n \neq 0 \\ \pi, & m=n=0 .\end{cases}
$$

We observe that the Chebyshev polynomials form an orthogonal set on the interval $[-1,1]$ with the weighting function $\frac{1}{\sqrt{1-x^{2}}}$.

\subsection{High order derivatives of Chebyshev polynomials}

(The Leibniz Formula) For a function $f(x)=g(x) h(x)$, the derivatives of $f(x)$ can be represented as a sum of derivatives of $g(x)$ and $h(x)$ as:

$$
f^{(k)}(x)=\sum_{n=0}^{k}\left(\begin{array}{l}
k \\
n
\end{array}\right) g^{(n)}(x) h^{(k-n)}(x),
$$

where $\left(\begin{array}{l}k \\ n\end{array}\right)$ are the binomial coefficients.

Theorem 2.1. (Slevinsky-Safouhi)[54] Let $G(x)$ be a function kth differentiable and with the term $\left(\frac{d}{x d x}\right)^{k} G(x)$ welldefined. The term $\frac{d^{k} G}{d x^{k}}$ is given by:

$$
\frac{d^{k} G}{d x^{k}}=\sum_{i=\left\lfloor\frac{k+1}{2}\right\rfloor}^{k} \hat{A}_{k}^{i} x^{2 i-k}\left(\frac{d}{x d x}\right)^{k} G(x),
$$

with coefficients:

$$
\hat{A}_{k}^{i}= \begin{cases}1, & i=k ; \\ 2 \hat{A}_{k-1}^{i}+\hat{A}_{k-1}^{i-1}, & i=\left\lfloor\frac{k+1}{2}\right\rfloor, k \text { odd } ; \\ \hat{A}_{k-1}^{i}, & i=\left\lfloor\frac{k+1}{2}\right\rfloor, k \text { even } \\ (2 i-k+1) \hat{A}_{k-1}^{i}+\hat{A}_{k-1}^{i-1}, & \left\lfloor\frac{k+1}{2}\right\rfloor<i<k, k>3 .\end{cases}
$$

where $\lfloor\alpha\rfloor$ is the integer floor function of argument $\alpha$.

It is natural with the help of the Leibniz Formula as well as Rodrigue's formula to define the higher order derivatives of $T_{n}(x)$ as:

$$
\begin{aligned}
\frac{\mathrm{d}^{i}}{\mathrm{~d} x^{i}} T_{n}(x)= & \frac{\Gamma\left(\frac{1}{2}\right)}{(-2)^{n} \Gamma\left(n+\frac{1}{2}\right)} \sum_{l=0}^{i}\left(\begin{array}{l}
i \\
l
\end{array}\right) \frac{\mathrm{d}^{l}}{\mathrm{~d} x^{l}} \sqrt{1-x^{2}} \\
& \frac{\mathrm{d}^{n+i-l}}{\mathrm{~d} x^{n+i-l}}\left(1-x^{2}\right)^{n-\frac{1}{2}} .
\end{aligned}
$$

Without going into great detail, if we apply the result of Theorem 2.1 into the above equation then we develop a very effective formula as the final result [54]:

$$
\begin{aligned}
& \frac{\mathrm{d}^{k}}{\mathrm{~d} x^{k}} T_{n}(x)=\frac{\Gamma\left(\frac{1}{2}\right)}{(-2)^{n} \Gamma\left(n+\frac{1}{2}\right)} \\
& \sum_{l=0}^{k}\left\{\left(\begin{array}{l}
k \\
l
\end{array}\right)\left[\sum_{i=\left\lfloor\frac{l+1}{2}\right\rfloor}^{l} \hat{A}_{l}^{i} x^{2 i-l}(-2)^{i}\left(1-x^{2}\right)^{\frac{1}{2}-i} \prod_{j=0}^{i-1}\left(\frac{1}{2}-j\right)\right]\right.
\end{aligned}
$$$$
\left.\left[\sum_{i=\left\lfloor\frac{n+k-l+1}{2}\right\rfloor}^{n+k-l} \hat{A}_{n+k-l}^{i} x^{2 i-n-k+l}(-2)^{i}\left(1-x^{2}\right)^{n-\frac{1}{2}-i} \prod_{j=0}^{i-1}\left(n-\frac{1}{2}-j\right)\right]\right\}
$$

with coefficients $\hat{A}_{k}^{i}$ given by (14).

\section{Nonlinear optimization model}

By the change of variable $x \mapsto \frac{1}{2}(x+1)$, the boundary value problems (2)-(3) and (2)-(4) can be rewritten as

$$
(1-x) \frac{\mathrm{d}^{2} \theta}{\mathrm{d} x^{2}}-\frac{\mathrm{d} \theta}{\mathrm{d} x}=\frac{1}{2}\left[\alpha^{2} B i\left(\theta-\theta_{\infty}\right)+\alpha^{2} N_{r}\left(\theta^{4}-\theta_{s}^{4}\right)\right],
$$

$$
\theta(-1)=1, \theta^{\prime}(1)=0,
$$

and

$$
\begin{aligned}
\left(3-x^{2}-2 x\right) \frac{\mathrm{d}^{2} \theta}{\mathrm{d} x^{2}}-2(1+x) \frac{\mathrm{d} \theta}{\mathrm{d} x}= & \alpha^{2} B i\left(\theta-\theta_{\infty}\right) \\
& +\alpha^{2} N_{r}\left(\theta^{4}-\theta_{s}^{4}\right),
\end{aligned}
$$

$$
\theta(-1)=1, \theta^{\prime}(1)=0,
$$

respectively. Now, it is convenient to treat them by Chebyshev polynomials of the first kind. Moreover, the change of function $\theta \mapsto \theta+1$ transforms the problems into

$$
\begin{aligned}
& (1-x) \frac{\mathrm{d}^{2} \theta}{\mathrm{d} x^{2}}-\frac{\mathrm{d} \theta}{\mathrm{d} x} \\
& =\frac{1}{2}\left[\alpha^{2} B i\left(1+\theta-\theta_{\infty}\right)+\alpha^{2} N_{r}\left((1+\theta)^{4}-\theta_{s}^{4}\right)\right],
\end{aligned}
$$

$$
\theta(-1)=0, \theta^{\prime}(1)=0 \text {, }
$$

and

$$
\begin{gathered}
\left(3-x^{2}-2 x\right) \frac{\mathrm{d}^{2} \theta}{\mathrm{d} x^{2}}-2(1+x) \frac{\mathrm{d} \theta}{\mathrm{d} x}= \\
\alpha^{2} B i\left(1+\theta-\theta_{\infty}\right) \\
+\alpha^{2} N_{r}\left((1+\theta)^{4}-\theta_{s}^{4}\right), \\
\theta(-1)=0, \theta^{\prime}(1)=0,
\end{gathered}
$$

such that the boundary conditions become homogenous. 


\subsection{Reformed version of Chebyshev polynomials}

Define $\hat{T}_{n}, n \geq 1$ as

$$
\hat{T}_{n}(x)=T_{n}(x)-n^{2} x-n^{2}-(-1)^{n}, n \geq 1,
$$

then obviously, from (10), we have

$$
\hat{T}_{n}(-1)=0, n \geq 1 \text {. }
$$

Eq. (9) implies

$$
\hat{T}_{n}^{\prime}(1)=T_{n}^{\prime}(1)-n^{2}=0 \quad n \geq 1 .
$$

Therefore, from Eqs. (26)-(27), we conclude that the boundary conditions (22) and (24) hold.

Furthermore, the second derivative of the reformed version of Chebyshev polynomials of the first kind are given by

$$
\begin{aligned}
& \hat{T}_{n}^{\prime}(x)=T_{n}^{\prime}(x)-n^{2}, \quad n \geq 1, \\
& \hat{T}_{n}^{\prime \prime}(x)=T_{n}^{\prime \prime}(x), \quad n \geq 1,
\end{aligned}
$$

where the right hand side can be obtained by the formula (16) when $k=1,2$.

\subsection{Corresponding optimization problem}

Define a approximate series solution of order $M$ as

$$
\Theta_{M}(x)=\sum_{n=1}^{M} \alpha_{n} \hat{T}_{n}(x),
$$

and consider the number of $N$ regularly distributed nodal points in interval $[-1,1]$, namely $x_{i}, i=1,2, \ldots, N$, then we define the unsupervised errors as the sum of mean squared errors:

$$
\begin{aligned}
& \epsilon_{1}(N, \alpha)=\frac{1}{N} \sum_{i=1}^{N}\left\{\left(1-x_{i}\right) \sum_{n=1}^{M} \alpha_{n} \hat{T}_{n}^{\prime \prime}\left(x_{i}\right)-\sum_{n=1}^{M} \alpha_{n} \hat{T}_{n}^{\prime}\left(x_{i}\right)-\right. \\
& \frac{1}{2}\left[\alpha^{2} B i\left(1+\sum_{n=1}^{M} \alpha_{n} \hat{T}_{n}\left(x_{i}\right)-\theta_{\infty}\right)\right. \\
& \left.\left.+\alpha^{2} N_{r}\left(\left(1+\sum_{n=1}^{M} \alpha_{n} \hat{T}_{n}\left(x_{i}\right)\right)^{4}-\theta_{s}^{4}\right)\right]\right\}^{2}
\end{aligned}
$$

for triangular fin, and

$$
\begin{aligned}
& \epsilon_{2}(N, \alpha)=\frac{1}{N} \sum_{i=1}^{N}\left\{\left(3-x_{i}^{2}-2 x_{i}\right) \sum_{n=1}^{M} \alpha_{n} \hat{T}_{n}^{\prime \prime}\left(x_{i}\right)\right. \\
& -2\left(1+x_{i}\right) \sum_{n=1}^{M} \alpha_{n} \hat{T}_{n}^{\prime}\left(x_{i}\right)-\left[\alpha^{2} B i\left(1+\sum_{n=1}^{M} \alpha_{n} \hat{T}_{n}\left(x_{i}\right)-\theta_{\infty}\right)\right. \\
& \left.\left.+\alpha^{2} N_{r}\left(\left(1+\sum_{n=1}^{M} \alpha_{n} \hat{T}_{n}\left(x_{i}\right)\right)^{4}-\theta_{s}^{4}\right)\right]\right\}^{2},
\end{aligned}
$$

for concave parabolic fin. It is worth to mention here that $\Theta_{M}(x)$ automatically satisfy boundary conditions (22) and (24). Now, define the following optimization problems

$$
\begin{aligned}
& \min _{\alpha} \epsilon_{1}(N, \alpha) \\
& \text { subject to } \epsilon_{1}(N, \alpha)-\varepsilon \leq 0, \\
& \min _{\alpha} \epsilon_{2}(N, \alpha) \\
& \text { subject to } \epsilon_{2}(N, \alpha)-\varepsilon \leq 0,
\end{aligned}
$$

for triangular and concave parabolic fins, respectively, where $\varepsilon$ is a given tolerance. In our approach, the interior point method (IPM) is used for tuning of weights of the approximate series solution (30). IPM belongs to a class of algorithms which are used for treating constrained optimization problems. The technique is based on Karmarkar's algorithm which has been developed by Narendra Karmarkar in 1984 for linear programming resolution [55]. Detailed in formation about the algorithm is available in references [56, 57]. IPMs have been applied to many optimization problems in engineering and applied science such as multi-area optimal reactive power flow [58] and economic dispatch problem [59]. The fundamental trait of interior point methods are based on self-concordant barrier functions which play important role in encoding the convex set. In contrast to the classical simplex method, search for an optimal solution is made by traversing the interior of the feasible region and solving a sequence of subproblems [60].

\section{Numerical experiments and comparison}

In this section, we show the results obtained for some case studies which have been adopted from Refs. [2, 48-51] using proposed method described in the previous sections. In these examples, $N=30$, the number of total nodal points covering $[-1,1]$, is regularly distributed. Moreover, the number of basis function in approximate series solution in Eq. (30) is $M=10$. The obtained solutions can be compared to those of Refs. [2, 48-51] and references therein. All approximate solutions reported here obtained in seconds by MATLAB softwares programm, therefore the method is highly robust.

MATLAB provides an efficient optimization toolbox that contains functions for finding minimum of a multivariable function while satisfying constraints. The toolbox includes solvers that perform optimization on the various types of linear or nonlinear problems. The function, 
fmincon( $(\cdot)$, of this toolbox is a general, multipurpose optimizer that well tested and frequently used to solve nonlinear programming problems with general equality, inequality, and bound constraints of small, medium, and large scale. To handle optimization problem (33)-(34), we use fmincon( $(\cdot)$ augmented to the interior point method (IPM) as described in the previous section.

Figures $1-3$ show temperature distribution versus $x$ for different values of Biot number $B i=0.01,0.05,0.1,0.5,1,5,10$ and $\alpha=1,4,10$ and $N_{r}=0,0.1,1$ when $\theta_{s}=\theta_{\infty}=0.2$ in the case of triangular fin. The same graphs for the case of concave parabolic fin are plotted in Figures 4-6.

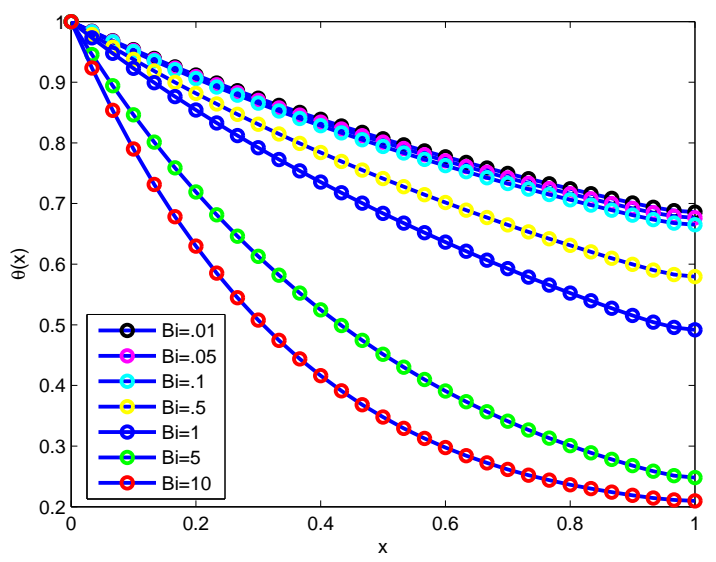

Figure 1: Diagram of temperature distribution versus $x$ for the triangular fin with $\alpha=1$ and $N_{r}=1$.

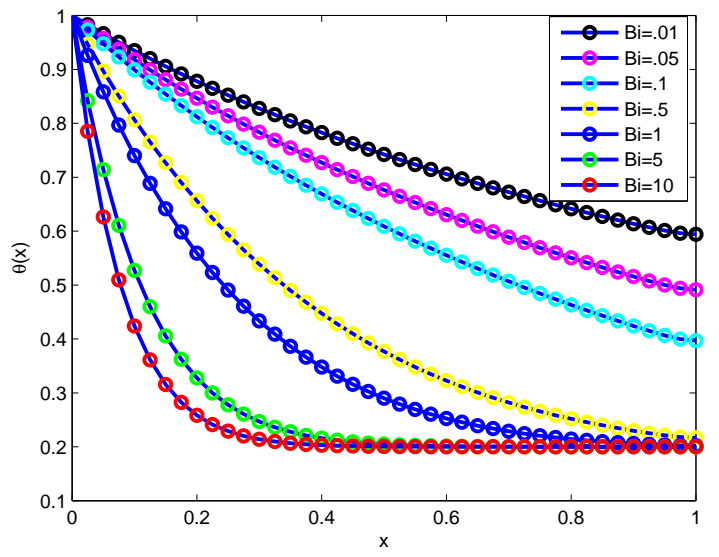

Figure 2: Diagram of temperature distribution versus $x$ for the triangular fin with $\alpha=4$ and $N_{r}=0.1$.

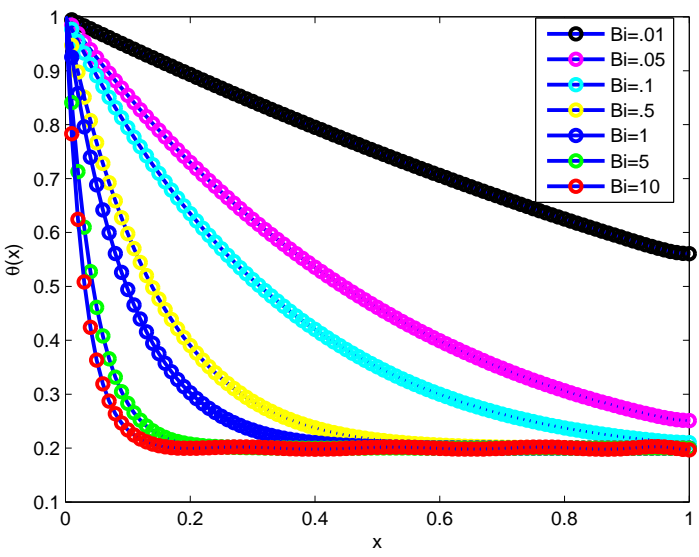

Figure 3: Diagram of temperature distribution versus $x$ for the triangular fin with $\alpha=10$ and $N_{r}=0$.

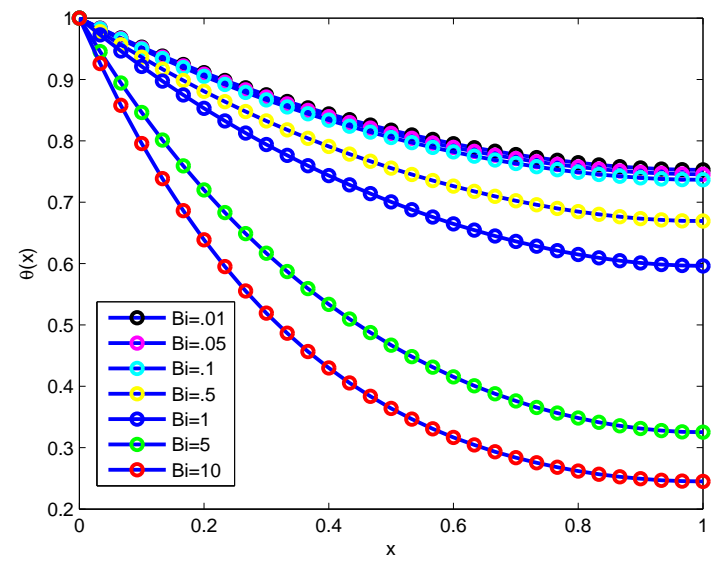

Figure 4: Diagram of temperature distribution versus $x$ for the concave parabolic fin with $\alpha=1$ and $N_{r}=1$.

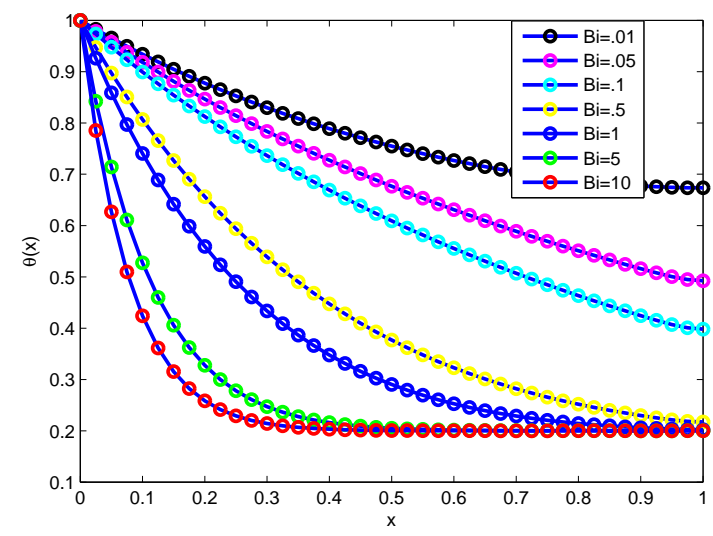

Figure 5: Diagram of temperature distribution versus $x$ for the concave parabolic fin with $\alpha=4$ and $N_{r}=0.1$. 


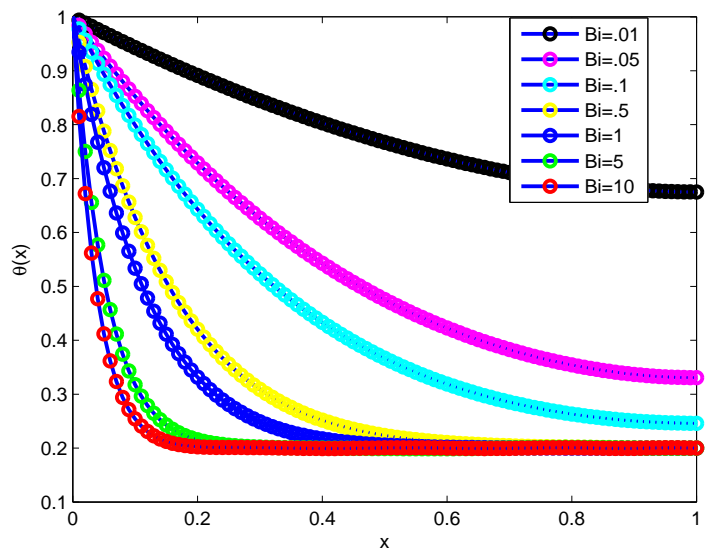

Figure 6: Diagram of temperature distribution versus $x$ for the concave parabolic fin with $\alpha=10$ and $N_{r}=0$.

The heat transfer rate $q$ (per unit depth) is given by

$$
q=-k w_{b} \frac{\mathrm{d} T(0)}{\mathrm{d} X}
$$

which is in dimensionless form as

$$
Q=\frac{q}{k T_{b}}=-\frac{1}{\alpha} \frac{\mathrm{d} \theta(0)}{\mathrm{d} x} .
$$

Fin efficiency is the ratio of the real heat transfer rate to the ideal heat transfer rate for a fin of infinite thermal conductivity

$$
\eta=\frac{q}{h\left(2 L+w_{t}\right)\left(T_{b}-T_{\infty}\right)+\left(2 L+w_{t}\right) \varepsilon \sigma\left(T_{b}^{4}-T_{s}^{4}\right)},
$$

which can be rewritten in dimensionless form as

$$
\eta=\frac{Q}{2(\alpha+1)\left[B i\left(1-\theta_{\infty}\right)+N_{r}\left(1-\theta_{s}^{4}\right)\right]},
$$

We have reported dimensionless heat transfer rate and the fin efficiency in the cases of the triangular and concave parabolic fin for different Biot number, radiationconduction number and $\alpha$ in Tables 1-4 when $\theta_{s}=\theta_{\infty}=$ 0.2 .

To validate our results, consider the boundary value problem (2)-(4) in the case $B i\left(1-\theta_{\infty}\right)=N_{r}\left(\theta_{S}^{4}-1\right)$, then it is easy to see that the unique solution to the above problem is $\theta(x) \equiv 1$ in both cases, triangular and concave parabolic fins. This point is in full agreement with our approximation results shown in Figs. 7 and 8 for any values satisfying $\operatorname{Bi}\left(1-\theta_{\infty}\right)=N_{r}\left(\theta_{s}^{4}-1\right)$.

\section{Conclusions}

In this article, the problem of the evaluation of heat transfer rate from convecting-radiating fin in the cases of triangular and concave parabolic shapes has been investigated.
Table 1: Dimensionless heat transfer rate for the triangular fin with $\theta_{s}=\theta_{\infty}=0.2$.

\begin{tabular}{cccc}
\hline$B i$ & $\alpha=1, N_{r}=1$ & $\alpha=4, N_{r}=0.1$ & $\alpha=10, N_{r}=0$ \\
\hline 0.01 & 0.48333 & 0.17135 & 0.05572 \\
0.05 & 0.49763 & 0.21644 & 0.15614 \\
0.1 & 0.51538 & 0.26672 & 0.22919 \\
0.5 & 0.65161 & 0.53104 & 0.52836 \\
1 & 0.80703 & 0.73927 & 0.74467 \\
5 & 1.65183 & 1.57822 & 1.59101 \\
10 & 2.31777 & 2.14760 & 2.16689 \\
\hline
\end{tabular}

Table 2: Fin efficiency for the triangular fin with $\theta_{s}=\theta_{\infty}=0.2$.

\begin{tabular}{cccc}
\hline$B i$ & $\alpha=1, N_{r}=1$ & $\alpha=4, N_{r}=0.1$ & $\alpha=10, N_{r}=0$ \\
\hline 0.01 & 0.12006 & 0.15889 & 0.31659 \\
0.05 & 0.11981 & 0.15478 & 0.17743 \\
0.1 & 0.11948 & 0.14831 & 0.13022 \\
0.5 & 0.11649 & 0.10624 & 0.06004 \\
1 & 0.11219 & 0.08156 & 0.04231 \\
5 & 0.08262 & 0.03849 & 0.01808 \\
10 & 0.06439 & 0.02651 & 0.01231 \\
\hline
\end{tabular}

Table 3: Dimensionless heat transfer rate for the concave parabolic fin with $\theta_{s}=\theta_{\infty}=0.2$.

\begin{tabular}{cccc}
\hline$B i$ & $\alpha=1, N_{r}=1$ & $\alpha=4, N_{r}=0.1$ & $\alpha=10, N_{r}=0$ \\
\hline 0.01 & 0.50120 & 0.17525 & 0.05939 \\
0.05 & 0.51591 & 0.22097 & 0.15904 \\
0.1 & 0.53416 & 0.27154 & 0.22319 \\
0.5 & 0.67405 & 0.51838 & 0.47271 \\
1 & 0.83310 & 0.69683 & 0.65199 \\
5 & 1.65707 & 1.40174 & 1.36063 \\
10 & 2.24615 & 1.88566 & 1.84815 \\
\hline
\end{tabular}

Table 4: Fin efficiency for the concave parabolic fin with $\theta_{s}=\theta_{\infty}=$ 0.2 .

\begin{tabular}{cccc}
\hline$B i$ & $\alpha=1, N_{r}=1$ & $\alpha=4, N_{r}=0.1$ & $\alpha=10, N_{r}=0$ \\
\hline 0.01 & 0.12450 & 0.16251 & 0.33744 \\
0.05 & 0.12421 & 0.15802 & 0.18073 \\
0.1 & 0.12383 & 0.15099 & 0.12681 \\
0.5 & 0.12050 & 0.10371 & 0.05372 \\
1 & 0.11581 & 0.07744 & 0.03704 \\
5 & 0.08288 & 0.03419 & 0.01546 \\
10 & 0.06240 & 0.02328 & 0.01050 \\
\hline
\end{tabular}




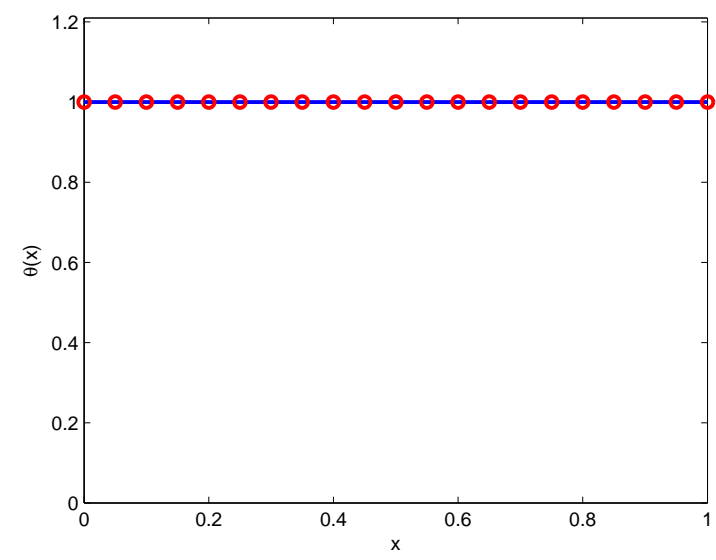

Figure 7: Diagram of temperature distribution versus $x$ for the triangular fin when $B i\left(1-\theta_{\infty}\right)=N_{r}\left(\theta_{s}^{4}-1\right)$.

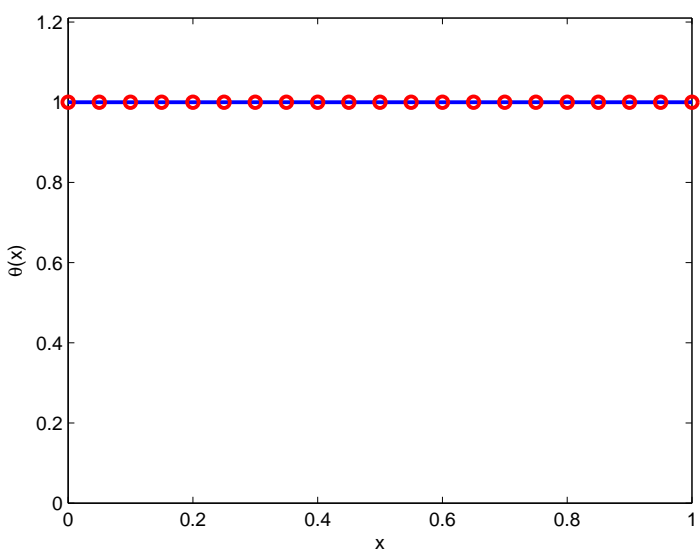

Figure 8: Diagram of temperature distribution versus $x$ for the concave parabolic fin when $\operatorname{Bi}\left(1-\theta_{\infty}\right)=N_{r}\left(\theta_{S}^{4}-1\right)$.

We have considered one-dimensional, steady conduction in the fin and neglected radiative exchange between adjacent fins and its primary surface.

It has been proposed a new intelligent computational technique to obtain approximate solution for the mentioned problem. First, the governing equation is transformed into an equivalent problem whose boundary conditions are homogeneous in interval $[-1,1]$. Then, it is optimized Chebyshev polynomials of the first kind to construct approximate series solution with unknown weights. Furthermore, by defining an optimization problem and minimizing it, all weights are obtained via interior point method. As a result, we have reported heat transfer rate and the fin efficiency in the cases of the triangular and concave parabolic fin for different Biot number and radiationconduction number with desired order of accuracy.
The method includes three steps: The first and most important step is to find Reformed Version of Chebyshev Polynomials i.e. Eq. (25) so that they satisfy the boundary conditions. The second step is to construct the optimizations problems (33) or (34) and the final step is to demand fmincon( $(\cdot)$ augmented to interior point algorithm using MATLAB. It has been revealed through test studies that the method is highly robust and reliable.

Acknowledgement: The authors would like to thank two anonymous referees for their valuable comments and helpful suggestions which have improved the quality of the paper.

\begin{tabular}{ll}
\hline Nomenclature & \\
$T$ & temperature \\
$T_{b}$ & fin base temperature \\
$T_{S}$ & effective sink temperature for radiation \\
$T_{\infty}$ & environment temperature for convection \\
$L$ & fin length \\
$h$ & convective heat transfer coefficient \\
$k$ & thermal conductivity \\
$N_{r}$ & radiation-conduction number \\
$X$ & dimensional space coordinate \\
$q$ & heat transfer rate \\
$Q$ & dimensionless heat transfer rate \\
$\chi$ & non-dimensional space coordinate \\
$w_{b}$ & fin thickness at the base \\
$w_{t}$ & fin thickness at the tip \\
$B i$ & Biot number \\
$w(x)$ & Profile function \\
$T_{n}(x)$ & n'th Chebyshev polynomials of the first kind \\
$\epsilon_{i}(N, \alpha)$ & Objective function in optimization model \\
Greeks symbols & \\
$\sigma$ & Stefan-Boltzmann constant \\
$\eta$ & fin efficiency \\
$\alpha$ & ratio of length to one-half base thickness \\
$\varepsilon$ & surface emissivity \\
$\theta$ & dimensionless temperature \\
$\theta_{S}$ & dimensionless effective sink temperature for radiation \\
$\theta_{\infty}$ & dimensionless environment temperature for convection \\
\hline &
\end{tabular}

\section{References}

[1] Kraus A.D., Sixty-five years of extended surface technology 1922-1987, Appl. Mech. Rev., 1988, 41(9), 321.

[2] Nguyen H., Aziz A., Heat transfer from convecting-radiating fins of different profile shapes, Int. J. Heat Mass Transf, 1992, 27(2), 67-72.

[3] Kraus A.D., Aziz A., Welty J., Extended Surface Heat Transfer, 2002, New York, John Wiley \& Sons.

[4] Cuce E., Cuce P.M., A successful application of homotopy perturbation method for efficiency and effectiveness assessment of longitudinal porous fins, Ener. Conv. Manag., 2015, 93, 9299.

[5] Cuce E., Cuce P.M., Homotopy perturbation method for temperature distribution, fin efficiency and fin effectiveness of 
convective straight fins with temperature-dependent thermal conductivity, Proc Inst Mech Eng Part C, J Mech Eng Sci, 2013, 227(8), 1754-1760.

[6] Ellahi R., Shivanian E., Abbasbandy S., Hayat T., Numerical study of magnetohydrodynamics generalized Couette flow of Eyring-Powell fluid with heat transfer and slip condition, Int. J. Numer. Meth. Heat Fluid Flow, 2016, 26(5), 1433-1445.

[7] Shirvan K.M., Mamourian M., Mirzakhanlari S., Ellahi R., Two phase simulation and sensitivity analysis of effective parameters on combined heat transfer and pressure drop in a solar heat exchanger filled with nanofluid by RSM, J. Mol. Liq., 2016, 220, 888-901.

[8] Mamourian M., Shirvan K.M., Ellahi R., Rahimi A., Optimization of mixed convection heat transfer with entropy generation in a wavy surface square lid-driven cavity by means of Taguchi approach, Int. J. Heat Mass Transf., 2016, 102, 544-554.

[9] Bhatti M., Zeeshan A., Ellahi R., Study of heat transfer with nonlinear thermal radiation on sinusoidal motion of magnetic solid particles in a dusty fluid, J. Theor. Appl. Mech., 2016, 46(3), 75-94.

[10] Shirvan K.M., Ellahi R., Mirzakhanlari S., Mamourian M., Enhancement of heat transfer and heat exchanger effectiveness in a double pipe heat exchanger filled with porous media, numerical simulation and sensitivity analysis of turbulent fluid flow, Appl. Therm. Eng., 2016, 109, 761-774.

[11] Majeed A., Zeeshan A., Ellahi R., Unsteady ferromagnetic liquid flow and heat transfer analysis over a stretching sheet with the effect of dipole and prescribed heat flux, J. Mol. Liq., 2016, 223, 528-533.

[12] Bhatti M.M., Zeeshan A., Ellahi R., Heat transfer analysis on peristaltically induced motion of particlefluid suspension with variable viscosity, clot blood model, Comp. Meth. Progr. Biomed., 2016, 137, 115-124.

[13] Khan A., Usman H., Vafai K., Ellahi R., Study of peristaltic flow of magnetohydrodynamic Walter's B fluid with slip and heat transfer, Scientia Iranica, 2016, 23(6), 2650-2662.

[14] Shirvan K.M., Mamourian M., Mirzakhanlari S., Ellahi R., Vafai K., Numerical investigation and sensitivity analysis of effective parameters on combined heat transfer performance in a porous solar cavity receiver by response surface methodology, Int. J. Heat Mass Transf., 2017, 105, 811-825.

[15] Rashidi S., Esfahani J.A., Ellahi R., Convective Heat Transfer and Particle Motion in an Obstructed Duct with Two Side by Side Obstacles by Means of DPMModel, Appl. Sci., 2017, 7(4), 431.

[16] Bhatti M., Zeeshan A., Ellahi R., Electromagnetohydrodynamic (EMHD) peristaltic flow of solid particles in a third-grade fluid with heat transfer, Mech. Industry, 2017, 18(3), 314.

[17] Cuce P.M., Cuce E., Effects of concavity level on heat loss, effectiveness and efficiency of a longitudinal fin exposed to natural convection and radiation, Int. J. Numer. Meth. Heat Fluid Flow, 2013, 23(7), 1169-1178.

[18] Arslanturk C., Analysis of thermal performance of annular fins with variable thermal conductivity by homotopy analysis method, Isi Bilimi ve Teknigi Dergisi, J. Therm. Sci. Technol., 2010, 30(2), 1-7.

[19] Turkyilmazoglu M., Exact solutions to heat transfer in straight fins of varying exponential shape having temperature dependent properties, Int. J. Therm. Sci., 2012, 55, 69-75.
[20] Ganji D., Rahimi M., Rahgoshay M., Determining the fin efficiency of convective straight fins with temperature dependent thermal conductivity by using homotopy perturbation method, Int. J. Numer. Meth. Heat Fluid Flow, 2012, 22(2), 263-272.

[21] Hatami M., Ganji D., Thermal performance of circular convective-radiative porous fins with different section shapes and materials, Ener. Conv. Manag., 2013, 76, 185-193.

[22] Torabi M., Zhang Q., Analytical solution for evaluating the thermal performance and efficiency of convective-radiative straight fins with various profiles and considering all nonlinearities, Ener. Conv. Manag., 2013, 66, 199-210.

[23] Hatami M., Hasanpour A., Ganji D., Heat transfer study through porous fins ( $\mathrm{Si} 3 \mathrm{~N} 4$ and $\mathrm{AL}$ ) with temperaturedependent heat generation, Ener. Conv. Manag., 2013, 74, 9-16.

[24] Campo A., Celentano D.J., Absolute maximum heat transfer rendered by straight fins with quarter circle profile using Finite Element Analysis, Appl. Therm. Eng., 2016, 105, 85-92.

[25] Unal H., An analytic study of boiling heat transfer from a fin, Int. J. Heat Mass Transf., 1987, 30(2), 341-349.

[26] Ghadikolaei S., Hosseinzadeh K., Yassari M., Sadeghi H., Ganji D., Boundary layer analysis of micropolar dusty fluid with $\mathrm{TiO} 2$ nanoparticles in a porous medium under the effect of magnetic field and thermal radiation over a stretching sheet, J. Mol. Liq., 2017, 244, 374-389.

[27] Ghadikolaei S., Yassari M., Sadeghi H., Hosseinzadeh K., Ganji D., Investigation on thermophysical properties of Tio2-Cu/H2O hybrid nanofluid transport dependent on shape factor in MHD stagnation point flow, Powder Technol., 2017, 322, 428-438.

[28] Ghadikolaei S., Hosseinzadeh K., Ganji D., Analysis of unsteady MHD Eyring-Powell squeezing flow in stretching channel with considering thermal radiation and Joule heating effect using AGM, Case Studies Thermal Eng., 2017, 10, 579-594.

[29] Hosseinzadeh K., Amiri A.J., Ardahaie S.S., Ganji D., Effect of variable lorentz forces on nanofluid flow in movable parallel plates utilizing analytical method, Case Studies Thermal Eng., 2017, 10, 595-610.

[30] Rahimi J., Ganji D., Khaki M., Hosseinzadeh K., Solution of the boundary layer flow of an Eyring- Powell non-Newtonian fluid over a linear stretching sheet by collocation method, Alexandria Eng. J., 2016.

[31] Dogonchi A., Hatami M., Hosseinzadeh K., Domairry G., Nonspherical particles sedimentation in an incompressible Newtonian medium by Pad'e approximation, Powder Technol., 2015, 278, 248- 256.

[32] Hatami M., Hosseinzadeh K., Domairry G., Behnamfar M., Numerical study of MHD two-phase Couette flow analysis for fluid-particle suspension between moving parallel plates, J. Taiwan Instit. Chem. Eng., 2014, 45(5), 2238-2245.

[33] Atouei S., Hosseinzadeh K., Hatami M., Ghasemi S.E., Sahebi S., Ganji D., Heat transfer study on convective-radiative semispherical fins with temperature-dependent properties and heat generation using efficient computational methods, Appl. Thermal Eng., 2015, 89, 299-305.

[34] Amiri A.J., Ardahaie S.S., Amooie A., Hosseinzadeh K., Ganji D., Investigating the effect of adding nanoparticles to the blood flow in presence of magnetic field in a porous blood arterial, Informat. Med. Unlocked, 2017.

[35] Sheikholeslami M., Ganji D.D., Moradi R., Forced convection in existence of Lorentz forces in a porous cavity with hot circular 
obstacle using nanofluid via Lattice Boltzmann method, J. Mol. Liq., 2017, 246, 103-111.

[36] Sheikholeslami M., Ganji D., Moradi R., Heat transfer of Fe304-water nanofluid in a permeable medium with thermal radiation in existence of constant heat flux, Chem. Eng. Sci., 2017, 174, 326-336.

[37] Sheikholeslami M., Nimafar M., Ganji D., Analytical approach for the effect of melting heat transfer on nanofluid heat transfer, Europ. Phys. J. Plus, 2017, 132(9), 385.

[38] Sheikholeslami M., Ganji D., Numerical analysis of nanofluid transportation in porous media under the influence of external magnetic source, J. Mol. Liq., 2017, 233, 499-507.

[39] Sheikholeslami M., Ziabakhsh Z., Ganji D., Transport of Magnetohydrodynamic nanofluid in a porous media, Colloids and Surfaces A, Physicochem. Eng. Aspects, 2017, 520, 201-212.

[40] Sheikholeslami M., Nimafar M., Ganji D., Nanofluid heat transfer between two pipes considering Brownian motion using AGM, Alexandria Eng. J., 2017, 56(2), 277-283.

[41] Sheikholeslami M., Ganji D.D., Impact of electric field on nanofluid forced convection heat transfer with considering variable properties, J. Mol. Liq., 2017, 229, 566-573.

[42] Sheikholeslami M., Ganji D., Free convection of Fe304-water nanofluid under the influence of an external magnetic source, J. Mol. Liq., 2017, 229, 530-540.

[43] Sheikholeslami M., Ganji D., Transportation of MHD nanofluid free convection in a porous semi annulus using numerical approach, Chem. Phys. Lett., 2017, 669, 202-210.

[44] Sheikholeslami M., Ganji D., Influence of magnetic field on $\mathrm{CuO}-\mathrm{H} 2 \mathrm{O}$ nanofluid flow considering Marangoni boundary layer, Int. J. Hydr. Ener., 2017, 42(5), 2748-2755.

[45] Mosayebidorcheh S., HatamiM,Mosayebidorcheh T., Ganji D., Optimization analysis of convective- radiative longitudinal fins with temperature-dependent properties and different section shapes and materials, Energy Conv. Manag., 2015, 106, 1286 1294.

[46] Ma J., Sun Y., Li B., Spectral collocation method for transient thermal analysis of coupled conductive, convective and radiative heat transfer in the moving plate with temperature dependent properties and heat generation, Int. J. Heat Mass Transf., 2017, 114, 469-482.
[47] Ma J., Sun Y., Li B., Simulation of combined conductive, convective and radiative heat transfer in moving irregular porous fins by spectral element method, Int. J. Thermal Sci., 2017, 118, 475-487.

[48] Bartas J., Sellers W., Radiation fin effectiveness, J. Heat Transfer, 1960, 82(1), 73-75.

[49] Singla R.K., Das R., Application of decomposition method and inverse prediction of parameters in a moving fin, Energy Conv. Manag., 2014, 84, 268-281.

[50] Singla R.K., Das R., et al, Application of decomposition method and inverse prediction of parameters in a moving fin (vol 84, pg 268, 2014), Energy Conv. Manag., 2015, 93, 458-459.

[51] Razelos P., Kakatsios X., Optimum dimensions of convectingradiating fins, Part I - longitudinal fins, Appl. Thermal Eng., 2000, 20(13), 1161-1192.

[52] Rivlin T., Chebyshev polynomials, 1990, New York, John Wiley \& Sons.

[53] Asmar N.H., Partial differential equations with Fourier series and boundary value problems, 2016, Courier Dover Publ.

[54] Slevinsky R.M., Safouhi H., New formulae for higher order derivatives and applications, J. Comp. Appl. Math., 2009, 233(2), 405-419.

[55] Karmarkar N., A new polynomial-time algorithm for linear programming, In: Proc. 16th An. ACM Symp. Theory Computing, ACM, 1984, 302-311.

[56] Wright S.J., Primal-dual interior-point methods, SIAM, 1997.

[57] Wright M., The interior-point revolution in optimization, history, recent developments, and lasting consequences, Bulletin Amer Math. Soc., 2005, 42(1), 39-56.

[58] Yan W., Wen L., Li W., Chung C., Wong K., Decompositioncoordination interior point method and its application to multi-area optimal reactive power flow, Int. J. Electr. Pow. Ener. Syst., 2011, 33(1), 55-60.

[59] Duvvuru N., Swarup K., A hybrid interior point assisted differential evolution algorithm for economic dispatch, IEEE Trans. Power Syst., 2011, 26(2), 541-549.

[60] Raja M.A.Z., Samar R., Numerical treatment for nonlinear MHD Jeffery-Hamel problem using neural networks optimized with interior point algorithm, Neurocomput., 2014, 124, 178-193. 